PROCEEDINGS OF THE

AMERICAN MATHEMATICAL SOCIETY

Volume 136, Number 11, November 2008, Pages 3809-3814

S 0002-9939(08)09421-5

Article electronically published on June 9, 2008

\title{
CRAMPED SUBGROUPS AND GENERALIZED HARISH-CHANDRA MODULES
}

\author{
BEN WEBSTER
}

(Communicated by Dan M. Barbasch)

\begin{abstract}
Let $G$ be a reductive complex Lie group with Lie algebra $\mathfrak{g}$. We call a subgroup $H \subset G$ cramped if there is an integer $b(G, H)$ such that each finite-dimensional representation of $G$ has a non-trivial invariant subspace of dimension less than $b(G, H)$. We show that a subgroup is cramped if and only if the moment map $T^{*}(K / L) \rightarrow \mathfrak{k}^{*}$ is surjective, where $K$ and $L$ are compact forms of $G$ and $H$. We will use this in conjunction with sufficient conditions for crampedness given by Willenbring and Zuckerman (2004) to prove a geometric proposition on the intersections between adjoint orbits and Killing orthogonals to subgroups.

We will also discuss applications of the techniques of symplectic geometry to the generalized Harish-Chandra modules introduced by Penkov and Zuckerman (2004), of which our results on crampedness are special cases.
\end{abstract}

\section{INTRODUCTION}

Throughout this paper, we fix an inclusion of semi-simple complex Lie groups $H \subset G$. We will assume throughout that $G$ and $H$ are connected. Further, we fix Cartan and Borel subgroups $T \subset B \subset G$, such that the intersection $B \cap H$ is a Borel subgroup for $H$. Let $K, L$ be the fixed points on $G$ and $H$, respectively, of the Cartan involution associated to $T \subset B$. These are maximal compact subgroups of $G$ and $H$, respectively, and $S=T \cap K$ is a maximal torus of $K$.

We let $\mathcal{X}_{+}^{G}$ and $\mathcal{X}_{+}^{H}$ be the cone of dominant integral weights of the respective groups. For $\lambda \in \mathcal{X}_{+}^{G}, \mu \in \mathcal{X}_{+}^{H}$, let $V_{\lambda}$ and $W_{\mu}$ be the corresponding finitedimensional irreducible representations.

For each weight $\lambda \in \mathcal{X}_{+}^{G}$, we can consider the representation $V_{\lambda}$ restricted to $H$. Let $b(\lambda)$ be the minimum among the dimensions of non-trivial $H$-invariant subspaces of $V_{\lambda}$, that is, the dimension of the smallest irreducible $H$-representation which appears as a subspace of $V_{\lambda}$. Then we have an invariant of the inclusion $H \hookrightarrow G$ given by

$$
b(G, H)=\sup \left\{b(\lambda) \mid \lambda \in \mathcal{X}_{+}^{G}\right\} .
$$

That is, $b(G, \mathfrak{h})$ is the smallest integer such that every non-trivial $G$-representation has a non-trivial $H$-invariant subspace $\leq b(G, \mathfrak{h})$.

Received by the editors December 16, 2006, and, in revised form, September 23, 2007.

2000 Mathematics Subject Classification. Primary 17B20; Secondary 53D20.

This material is based upon work supported under a National Science Foundation Graduate Research Fellowship and partially supported by the RTG grant DMS-0354321. 
Definition 1. We call the subgroup $H$ or corresponding inclusion cramped if $b(G, H)$ is finite.

An inclusion of Lie algebras $\mathfrak{h} \subset \mathfrak{g}$ will also be called cramped if the corresponding inclusion of groups $H \subset G$ where $G$ is simply-connected is cramped.

Note that the notion of crampedness requires a choice of a category of representations. By convention, we will consider the category of finite-dimensional complex algebraic representations if the group in question is complex reductive, and the category of finite-dimensional smooth complex representations if the group is compact. Since restriction from a complex reductive group to its maximal compact is an equivalence, $H \subset G$ is cramped over the category of complex algebraic representations if and only if $L \subset K$ is over the category of complex smooth representations.

In [WZ04, Prop. 3.5.1], Willenbring and Zuckerman prove the following theorem:

Theorem 1.1. If $G$ and $H$ are semi-simple, and $\operatorname{dim} H<\operatorname{dim} G / P$ for all parabolic subgroups $P$, then $H$ is cramped. In particular, if $\mathfrak{h} \cong \mathfrak{s l}_{2} \mathbb{C}$, then $H$ is cramped if and only if $\mathfrak{h}$ is not a summand of $\mathfrak{g}$.

In this paper, we will give necessary and sufficient conditions for an inclusion of reductive groups to be cramped, using the geometry of moment maps. Peculiarly, it is not clear to the author how these conditions imply Willenbring and Zuckerman's. Instead, we use these in conjunction with our results to prove a geometric result on the intersection of adjoint orbits with Killing orthogonals to certain subgroups. An independent proof of this proposition (Proposition 2.3) would show that our results imply those of Willenbring-Zuckerman.

\section{Moment maps And CRAmped Subgroups}

For all notation and basic notions of symplectic geometry, we refer the reader to the book of Ana Cannas da Silva [CdS01].

As usual, we consider $\mathfrak{k}^{*}$ as a Poisson manifold with the Kostant-Kirillov Poisson structure: if $X, Y \in \mathfrak{g}$, and we let $i_{X}: \mathfrak{k}^{*} \rightarrow \mathbb{R}$ be the map given by pairing with $X$, then by definition $\left\{i_{X}, i_{Y}\right\}=i_{[X, Y]}$. Extending by the Leibniz rule and continuity, we can take the Poisson bracket of any two functions. Note that the linear functions of $\mathfrak{k}$ form a Lie subalgebra of the space of all functions, which is isomorphic to $\mathfrak{k}$.

If $M$ is a symplectic (or more generally Poisson) manifold, a smooth map $\mu$ : $M \rightarrow \mathfrak{k}^{*}$ is called a moment map if it is a Poisson map. In particular, if $\mu$ is a moment map, the functions $\left\{\mu^{*} i_{X}\right\}_{X \in \mathfrak{k}}$ form a Lie subalgebra of functions under a Poisson bracket. After taking Hamiltonian vector fields, we obtain a map of Lie algebras from $\mathfrak{k}$ to vector fields on $M$. If $K$ is simply-connected, and $\mu$ proper, then this will necessarily integrate to an action of $K$ on $M$.

If $N$ is any manifold with a $K$-action, then $M=T^{*} N$ has a canonical symplectic structure, also equipped with a $K$-action. This $K$-action is Hamiltonian, that is, given by a moment map $\mu_{N}: M \rightarrow \mathfrak{k}^{*}$. We define $\mu_{N}$ as follows: for $n \in N, \xi \in T_{n}^{*} N$ and $X \in \mathfrak{k}$, then $\langle\mu(n, \xi), X\rangle=\left\langle\xi, X_{n}\right\rangle$, where $X_{n} \in T_{n} N$ is the vector given by differentiating the action of $G$ in the direction of $X$. Let im $N$ be the image of $\mu_{N}$.

We can use moment maps in representation theory to gain information using the philosophy of geometric quantization and geometric invariant theory (for a readable heuristic discussion of this principle, see [GLS96, §3.1]).

We will use a weak formulation of this connection: 
Theorem 2.1 (Kirwan-Ness MFK94]). Let $L \subset K$ be an inclusion of compact Lie groups. Let $\lambda$ be a dominant integral weight of $K$ and $\mu$ a dominant integral weight of $L$, and let $\mathcal{O}_{\lambda}, \mathcal{O}_{\mu}$ be the corresponding coadjoint orbits. Let $\pi: \mathfrak{k}^{*} \rightarrow \mathfrak{l}^{*}$ be the natural projection given by restriction. Then

$$
\operatorname{Hom}_{L}\left(W_{n \mu}, V_{n \lambda}\right) \neq\{0\}
$$

for some integer $n$ if and only if $\mathcal{O}_{\mu} \subset \pi\left(\mathcal{O}_{\lambda}\right)$.

This theorem will be the key to our understanding of $b(\mathfrak{g}, \mathfrak{h})$. We consider the $K$-space $N=K / L$.

Theorem 2.2. The following are equivalent:

(1) The subgroup $H$ is cramped.

(2) The map $\mu_{N}: T^{*} N \rightarrow \mathfrak{k}^{*}$ is surjective.

(3) For each maximal parabolic subgroup $P \subset G$, there exists $x \in \mathfrak{k}$ s.t. $(x, \mathfrak{l})=$ 0 and $\operatorname{Stab}_{K} x$ is conjugate to $P \cap K$.

Since $\operatorname{im} N=\operatorname{Ad}_{G}^{*} \cdot \mathfrak{h}^{\perp}$, an inclusion of reductive groups is cramped if and only if the corresponding inclusion of algebras is cramped.

Heuristically, we should consider $T^{*} N$ as a "semi-classical limit" of the $K$ representation $L^{2}(N)$, and im $N$ as a continuous analogue of the $K$-support of $L^{2}(N)$. By Frobenius reciprocity, that support is the same as the set of representations with non-trivial $L$-invariants. Thus, it is reasonable that $\mu_{N}$ being surjective reflects the fact that all representations are "close" to having $L$-invariants.

Let $X$ be a metric space with distance function $D: X \times X \rightarrow \mathbb{R}$. For any subsets $A, B \subset X$, let

$$
\delta(A, B)=\inf _{\substack{a \in A \\ b \in B}} D(a, b) .
$$

Proof. (1) $\Rightarrow(2)$ : Assume there exists $\lambda \notin \operatorname{im} N$. We can assume $\lambda$ is dominant, possibly after applying conjugation by an element of $K$. Since 0 not lying in $\pi\left(\mathcal{O}_{\lambda}\right)$ is an open condition on $\lambda$, we may furthermore assume that $m \lambda$ is integral for some $m$. Of course, $\pi\left(\mathcal{O}_{m \lambda}\right)$ also avoids 0 , so we may assume $\lambda$ is dominant and integral. If $\operatorname{Hom}_{L}\left(W_{\mu}, V_{m \lambda}\right) \neq 0$, then by Theorem 2.1 above, $\mu \in m \cdot \pi\left(\mathcal{O}_{\lambda}\right)$. By homogeneity of $\operatorname{im} N$,

$$
\delta(m \lambda, \operatorname{im} N)=\delta\left(m \cdot \pi\left(\mathcal{O}_{\lambda}\right), 0\right)=m \cdot \delta(\pi(K \cdot \lambda), 0) .
$$

By the Weyl dimension formula, the set $\mathcal{D}_{n}=\left\{\mu \in \mathcal{X}_{+}^{H} \mid \operatorname{dim} W_{\mu}<n\right\}$ is bounded for all integers $n$. Let $\eta_{n}=\sup _{\mu \in \mathcal{D}_{n}} \delta(\mu, 0)$. Thus for each $n$, there is another integer $N$ such that for $m>N$, the intersection $\mathcal{D}_{n} \cap\left(m \cdot \pi\left(\mathcal{O}_{\lambda}\right)\right)$ is empty. Thus, $V_{m \lambda}$ has no non-trivial $H$-invariant subspaces of dimension less than $n$ and $b(G, H)=\infty$.

$(2) \Rightarrow(3)$ : Consider the coadjoint orbit $\mathcal{O}$ through a point with stabilizer $P$, that is, the dual of an orbit passing though the center of the corresponding subalgebra $\mathfrak{p}$. Since $\mu_{N}$ is surjective, the preimage of this orbit is non-empty, and by the transitivity of the action of $K$ on $N$, this preimage intersects the fiber over $e L$, which is naturally identified with $\mathfrak{l}^{\perp}$, the annihilator of $\mathfrak{l}$ in $\mathfrak{k}^{*}$, or equivalently, under the Killing form on $\mathfrak{k}$. Thus there is a point in $\mathfrak{l}^{\perp}$ stabilized by a maximal parabolic subgroup conjugate to $P$.

$(3) \Rightarrow(1)$ : Since there is a unique orbit (up to scaling) corresponding to each maximal parabolic subgroup, $\mathcal{O}_{\omega_{i}}$ intersects $\mathfrak{l}^{\perp}$. Thus $0 \in \pi\left(\mathcal{O}_{\omega_{i}}\right)$, and by Theorem [2.1] we have $V_{m_{i} \omega_{i}}^{H} \neq 0$ for some $m_{i}$. The theorem now follows from the 
arguments given by Willenbring and Zuckerman in [WZ04, 3.3.1,4.0.10]. We sketch their argument for completeness: if $x \in V_{m_{i} \omega_{i}}^{H}$, then multiplication by $x$ defines an $H$-equivariant injection $V_{\lambda} \rightarrow V_{\lambda+m_{i} \omega_{i}}$ by embedding each representation in the coordinate ring of $G / U$ (where $U$ is the unipotent radical of $B$ ). Thus, if $\lambda\left(\alpha_{i}\right) \geq m_{i}$ for any $i$, then $V_{\lambda}$ has an $H$-invariant subspace isomorphic to $V_{\lambda^{\prime}}$ for some $\lambda^{\prime} \leq \lambda$. By induction,

$$
b(G, H)=\inf \left\{b(\lambda) \mid \lambda\left(\alpha_{i}^{\vee}\right)<m_{i}\right\}<\infty .
$$

Combining our results with those of Willenbring-Zuckerman, we obtain a seemingly new result on the geometry of Killing orthogonals to subgroups in semi-simple groups.

Proposition 2.3. Let $L \subset K$ be an inclusion of semi-simple, compact groups. If every $K$-orbit $\mathcal{O}$ on $\mathfrak{k}$ satisfies $\operatorname{dim} \mathcal{O}>2 \operatorname{dim} L$, then for each orbit $\mathcal{O}$, we have $\mathcal{O} \cap \mathfrak{l}^{\perp} \neq \emptyset$, where $\mathfrak{l}^{\perp}$ is the orthogonal complement to $\mathfrak{l}$ under the Killing form.

Proof. By [WZ04, Prop. 3.5.8], this inclusion $L \subset K$ is cramped since any adjoint orbit of minimal dimension is of the form $K / P \cap K$ for some maximal parabolic subgroup $P$ in the complexification $G$ of $K$. By Theorem 2.2 the crampedness of the inclusion $L \subset K$ is equivalent to the desired result.

If one could find an independent proof of this result, then it would directly show that the necessary and sufficient conditions of Theorem 2.2 imply Theorem 1.1. This seems to the author to be more elegant, but we have found no proof of this result notably simpler or shorter than the concatenation of Willenbring and Zuckerman's with ours.

\section{Generalized Harish-Chandra modules}

Fix an inclusion of reductive Lie algebras $\tilde{\mathfrak{g}} \supset \mathfrak{g}$. Following Penkov and Zuckerman [PZ04, we call a $\tilde{\mathfrak{g}}$-module a generalized Harish-Chandra module for the pair $(\tilde{\mathfrak{g}}, \mathfrak{g})$ if as a $\mathfrak{g}$-module if it is completely reducible with finite-dimensional isotypic components. Obviously, all finite-dimensional $\tilde{\mathfrak{g}}$-modules lie in this category, but there are also many infinite-dimensional ones which are quite mysterious.

The original inspiration for considering cramped subgroups derives from the theory of generalized Harish-Chandra modules. Let $\mathfrak{h} \subset \mathfrak{g}$ is a reductive subalgebra.

Proposition 3.1. If $\mathfrak{h}$ is cramped, then a generalized Harish-Chandra module $V$ for $(\tilde{\mathfrak{g}}, \mathfrak{g})$ is also a generalized Harish-Chandra module for $(\tilde{\mathfrak{g}}, \mathfrak{h})$ if and only if $V$ is finite-dimensional.

Proof. Obviously, $V$ is completely reducible as a $\mathfrak{h}$-module, and all irreducible summands are finite-dimensional, so $V$ is a generalized Harish-Chandra module if and only if all multiplicities of irreducible representations are finite. If $V$ is infinitedimensional, it has infinitely many isotypic components, and since $\mathfrak{h}$ is cramped, each of these has an irreducible, non-trivial, $\mathfrak{h}$-invariant subspace of dimension less than $b(\mathfrak{g}, \mathfrak{h})$. Since $\mathcal{D}_{n}$ is finite for all $n$, one of these representations has infinite multiplicity, and $V$ is not generalized Harish-Chandra.

Of course, the more general question arises: when is a $(\tilde{\mathfrak{g}}, \mathfrak{g})$ generalized HarishChandra module $V$ also a generalized Harish-Chandra module for the pair $(\tilde{\mathfrak{g}}, \mathfrak{h})$ if $H$ is not cramped? 
Using the techniques of symplectic geometry in the previous section, we will give a characterization of generalized Harish-Chandra modules for $(\tilde{\mathfrak{g}}, \mathfrak{g})$ which are also generalized Harish-Chandra for the pair $(\tilde{\mathfrak{g}}, \mathfrak{h})$ in terms of the image of the moment $\operatorname{map} \mu_{N}$.

Let $\operatorname{supp}_{\mathfrak{g}} V \subset \mathcal{X}_{+}^{\mathfrak{g}}$ be the $\mathfrak{g}$-support of $V$, that is, the set of weights $\lambda$ such that $\operatorname{Hom}_{\mathfrak{g}}\left(V_{\lambda}, V\right) \neq\{0\}$.

For each $\gamma \in \mathbb{R}_{\geq 0}$, let $\mathcal{N}_{\gamma}=\left\{\lambda \in \mathcal{X}_{+}^{\mathfrak{g}} \mid \delta(\lambda\right.$, im $\left.N) \leq \gamma\right\}$.

Theorem 3.2. $A(\tilde{\mathfrak{g}}, \mathfrak{g})$ generalized Harish-Chandra module $V$, is a generalized Harish-Chandra module for $(\tilde{\mathfrak{g}}, \mathfrak{h})$ if and only if for each $\gamma \in \mathbb{R}_{\geq 0}$, the intersection $\mathcal{N}_{\gamma} \cap \operatorname{supp}_{\mathfrak{g}} V$ is finite.

We will first prove a lemma which leads up to the proof of this theorem. Let $\mathcal{D}_{n}^{\prime}=\left\{\lambda \in \mathcal{X}_{+}^{\mathfrak{g}} \mid \operatorname{supp}_{H} V_{\lambda} \cap \mathcal{D}_{n} \neq \emptyset\right\}$.

Lemma 3.3. For each integer $n \in \mathbb{Z}$ and real number $\gamma$, there exists a real number $\beta \in \mathbb{R}_{\geq 0}$ and another integer $m \in \mathbb{Z}$, such that

$$
\begin{aligned}
& \mathcal{D}_{n}^{\prime} \subset \mathcal{N}_{\beta}, \\
& \mathcal{N}_{\gamma} \subset \mathcal{D}_{m}^{\prime} .
\end{aligned}
$$

Proof. If $\lambda \in \mathcal{D}_{n}^{\prime}$, then $\pi\left(\mathcal{O}_{\lambda}\right) \cap \mathcal{D}_{n} \neq \emptyset$. Applying $\pi$, we find that

$$
\delta(\lambda, \operatorname{im} N)=\delta\left(\pi\left(\mathcal{O}_{\lambda}\right), 0\right) \leq \eta_{n}
$$

That is, $\mathcal{D}_{n}^{\prime} \subset \mathcal{N}_{\eta_{n}}$.

Note that $\mathcal{D}_{1}^{\prime}$ is a subsemigroup of full rank in $\operatorname{im} N \cap \mathcal{X}_{+}^{\mathfrak{g}}$. Thus, for each point in $\lambda \in \mathcal{N}_{\gamma}$ sufficiently far from the origin, there is an element $\lambda^{\prime} \in \mathcal{D}_{1}^{\prime}$ such that $\lambda^{\prime \prime}=\lambda-\lambda^{\prime} \in \mathcal{N}_{\gamma}$. By definition, $\lambda^{\prime \prime}$ is smaller than $\lambda$ in the standard poset structure of $\mathcal{X}_{+}^{\mathfrak{g}}$. Thus, for some $\eta_{\gamma}$, we have

$$
m_{\gamma}=\sup _{\lambda \in \mathcal{N}_{\gamma}} b(\lambda)=\sup _{\substack{\lambda \in \mathcal{N}_{\gamma} \\ \delta(\lambda, 0)<\eta_{\gamma}}} b(\lambda)<\infty
$$

since a ball of radius $\eta_{\gamma}$ is compact. This is the desired integer $m$.

Proof of Theorem 3.2. The module $V$ is generalized Harish-Chandra if and only if the intersection $\mathcal{D}_{n}^{\prime} \cap \operatorname{supp}_{\mathfrak{g}} V$ is finite for each $n$. Since each set $\mathcal{N}_{\gamma}$ contains and is contained in a set of the form $\mathcal{D}_{n}^{\prime}$, these intersections are finite if and only if the intersections $\mathcal{N}_{\gamma} \cap \operatorname{supp}_{\mathfrak{g}} V$ are also finite.

Unfortunately, this is not a particularly easy criterion to apply, and we aim to simplify it. We can supply such a simplification, but at the price of putting a restriction on our representations. To do this, we need the notion of asymptotic support and vagrancy.

Let $\operatorname{asupp}_{\mathfrak{g}} V \subset \mathfrak{t}^{*}$ be the asymptotic support of $V$, that is, the points $\lambda \in \mathfrak{t}^{*}$ such that there is a sequence $a_{i} \in \mathbb{R}_{\geq 0}$ converging to 0 , and a sequence $\lambda_{i} \in \operatorname{supp}_{\mathfrak{g}} V$ such that $\lim _{x \rightarrow \infty} a_{i} \lambda_{i}=\lambda$.

Define the vagrancy of $V$ by

$$
\operatorname{vag}(V)=\sup _{\lambda \in \operatorname{supp}_{\mathfrak{g}} V} \delta\left(\lambda, \operatorname{asupp}_{\mathfrak{g}} V\right)+\sup _{\lambda \in \operatorname{asupp}_{\mathfrak{g}} V} \delta\left(\lambda, \operatorname{supp}_{\mathfrak{g}} V\right)
$$

Corollary 3.4. For all $(\tilde{\mathfrak{g}}, \mathfrak{g})$ generalized Harish-Chandra modules $V$ such that $\operatorname{vag}(V)<\infty$, the following are equivalent: 
(1) $\operatorname{im} N \cap \operatorname{asupp}_{\mathfrak{g}} V=\{0\}$.

(2) $V$ is generalized Harish-Chandra for $(\tilde{\mathfrak{g}}, \mathfrak{h})$.

Note that an essentially identical hypothesis, with similar conclusions, appears in the work of Kobayashi Kob02 in the much more difficult context of arbitrary unitary representations of real groups.

Proof. $(1) \Rightarrow(2)$ : By the definition of $\operatorname{vag}(V)$, the set $\operatorname{supp} V$ is contained in the set of elements of distance less than or equal to $\operatorname{vag}(V)$ from $\operatorname{asupp} V$. The intersection of this with $\mathcal{N}_{\gamma}$ for all $\gamma$ is compact (since it is closed and bounded), and thus has only finitely many elements of $\operatorname{supp} V$.

$(2) \Rightarrow(1)$ : By the definition of vag $(V)$, each ray in $\operatorname{im} N \cap \operatorname{asupp} V$ has infinitely many points of $\operatorname{supp} V$ within distance $\operatorname{vag}(V)$ from it, and thus $\mathcal{N}_{\operatorname{vag}(V)}$ contains infinitely many elements of $\operatorname{supp} V$.

As usual, this result raises more questions about the structure of Harish-Chandra modules than it answers. Foremost among them (in the author's opinion) is the mysterious property of finite vagrancy.

Question. Is vag $(V)$ always finite? Alternatively, is there a natural characterization of $V$ such that $\operatorname{vag}(V)$ is finite?

\section{ACKNOWLEDGMENTS}

The author would like to thank Noah Snyder and Ivan Penkov for useful discussions.

\section{REFERENCES}

[CdS01] Ana Cannas da Silva. Lectures on symplectic geometry, volume 1764 of Lecture Notes in Mathematics. Springer-Verlag, Berlin, 2001. MR1853077 (2002i:53105)

[GLS96] Victor Guillemin, Eugene Lerman, and Shlomo Sternberg. Symplectic fibrations and multiplicity diagrams. Cambridge University Press, Cambridge, 1996. MR:1414677 (98d:58074)

[Kob02] Toshiyuki Kobayashi, Branching problems of unitary representations, Proceedings of the ICM, Beijing, 2002, vol. 2, 615-627, Higher Ed. Press, Beijing, 2002. MR.1957069 $(2003 \mathrm{~m}: 22017)$

[MFK94] D. Mumford, J. Fogarty, and F. Kirwan. Geometric invariant theory, vol. 34 of Ergebnisse der Mathematik und ihrer Grenzgebiete (2) [Results in Mathematics and Related Areas (2)]. Springer-Verlag, Berlin, third edition, 1994. MR1304906 (95m:14012)

[PZ04] Ivan Penkov and Gregg Zuckerman. Generalized Harish-Chandra modules: A new direction in the structure theory of representations. Acta Appl. Math., 81(1-3):311-326, 2004. MR2069343 (2005d:17013)

[WZ04] Jeb F. Willenbring and Gregg Zuckerman. Small semisimple subalgebras of semisimple Lie algebras, 2004, arXiv:math.RT/0408302.

Department of Mathematics, University of California, Berkeley, Berkeley, CaliFORNIA 94720

Current address: School of Mathematics, Institute for Advanced Study, Princeton, New Jersey 09540

E-mail address: bwebste@ias.edu

URL: http://math.ias.edu/ ${ }^{\text {bwebste }}$ 